J. Product. \& Dev., 20(2): 149 - 159(2015)

تقدير دالة استجابة العرض لمحصول العنب فى ليبيا خلال القترة

$$
(r+1 \cdot-199 \cdot)
$$

ا.محمد يوسف بن عيسى

محاضر مساعد - جامعة طر ابلس ئس ئس
دـ.حمد المبروك الصادق

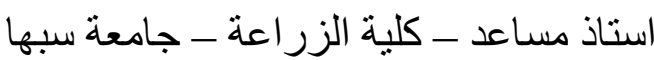

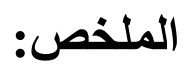

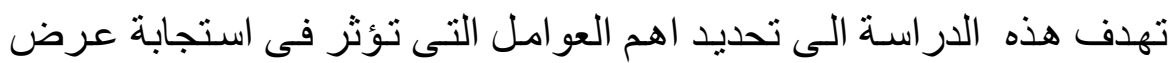

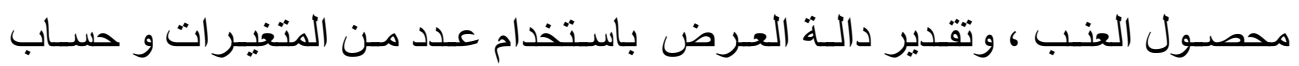

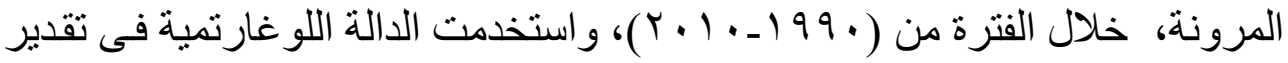

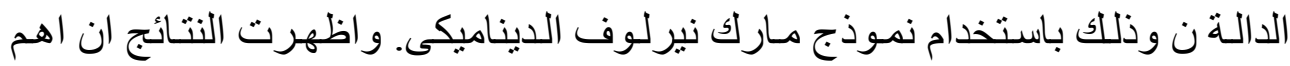

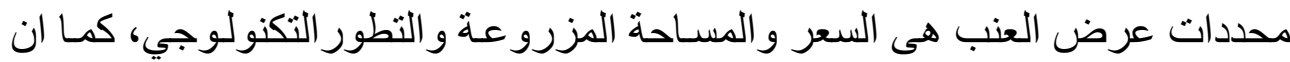

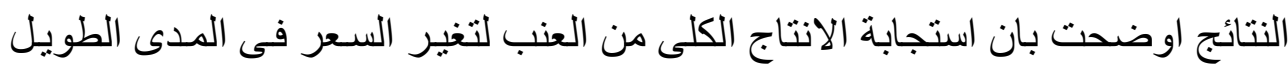
و القصير بانها غير مرنة.

المقدمة:

يعتبر محصول العنب من محاصيل الفاكهة الاساسية وتتفاوت الميزة النسبية فى

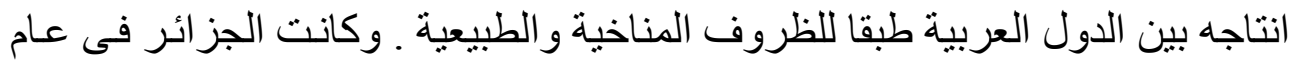

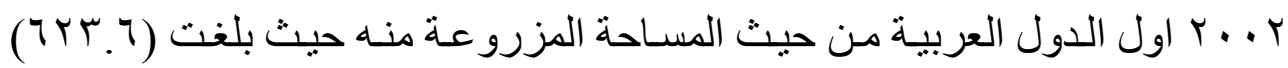

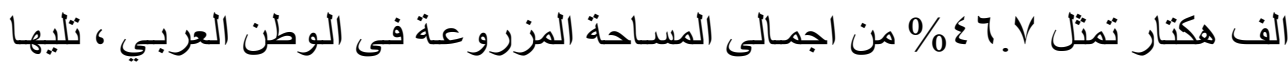

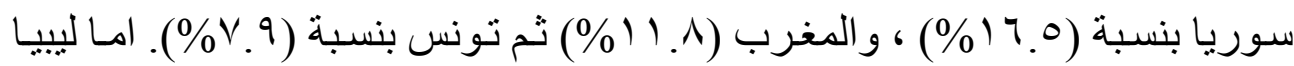

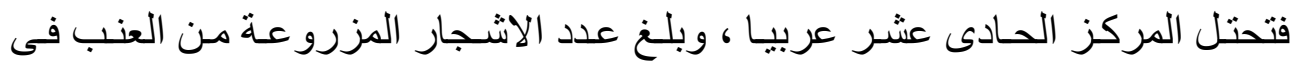

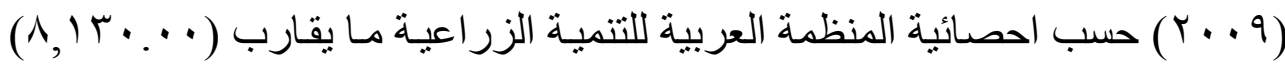

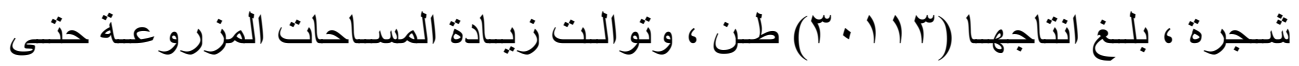

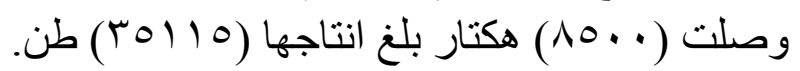

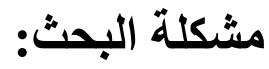

الاهمية الاقتصادية و الغذائية لهذا المحصول من المؤشرات التى تدفع باتجاه

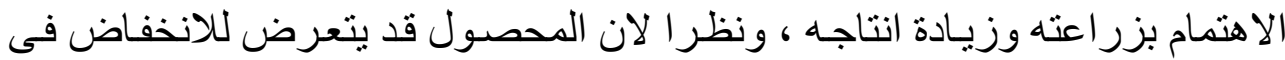

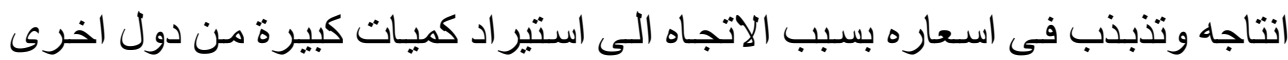
وكذلك تعرض الكثير من اصنافه في ليبيا الى الاصابة ببعض الامر اض النباتية و عدم التئ 
مكافحتها بالثكل المطلوب ادى الى انخفاض الانتاج. ولنقص الدراسـات الاقتصـادية

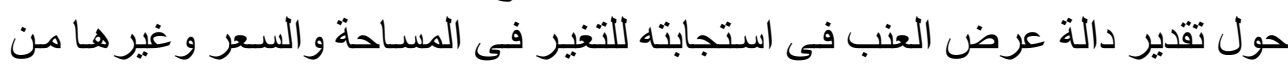

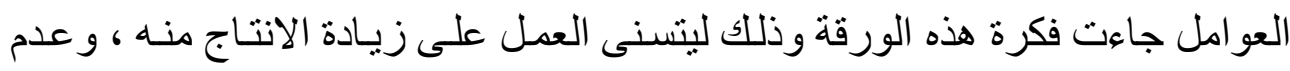
الاستير اد من الخارج خاصة وان النكرة الظروف المناخية ملائمة في ليبيا.

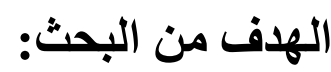

الهدف الرئيسي هو استخدام نتائج هذه الدراسة فى زيادة انتاج العنب واستخدام

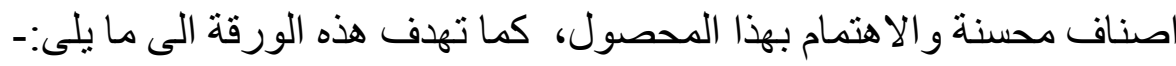

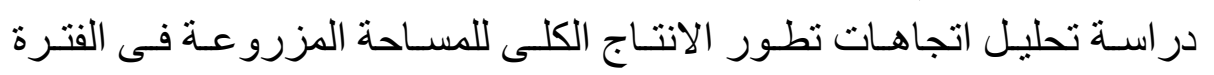
(Y.1.-199•)

تقدير دالة استجابة العنب باستخدام عدد من المتغير ات وحساب المرونة . تحديد اهم العوامل المؤثرة فى استجابة عرض العن العنب.

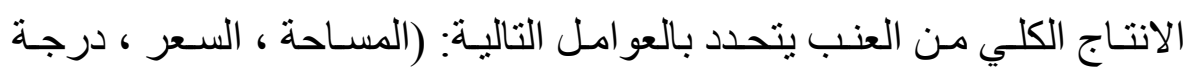
فرضية البحث:

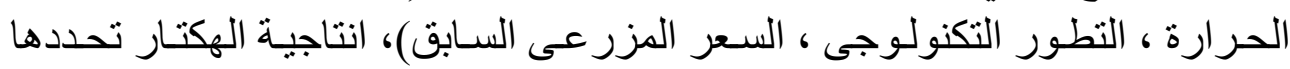

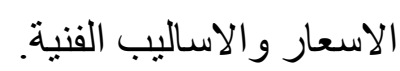
الحدود الزمنية ومصادر البيانات/

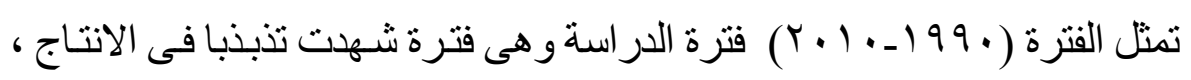
كما انها تعتبر مكملة لبعض الدر استات السابقة.

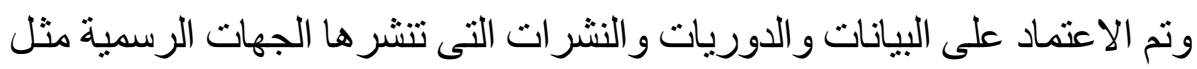

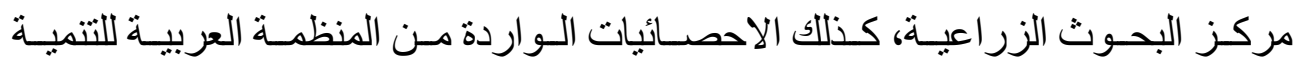
و الزر اعة و منظمة الاغذية والزر اعة (الفاو).

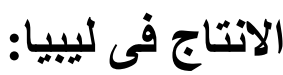

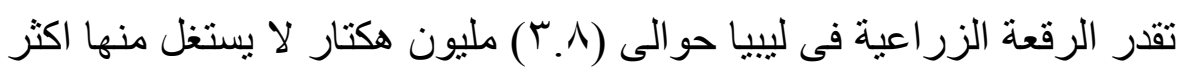

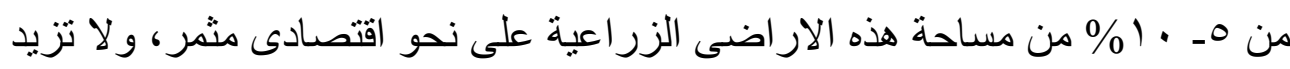

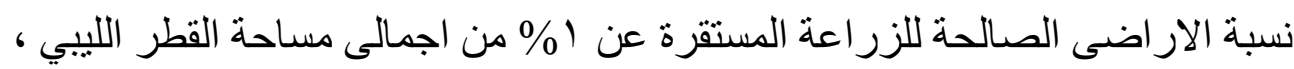

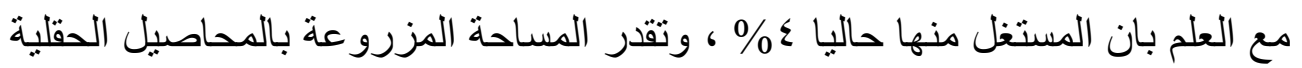

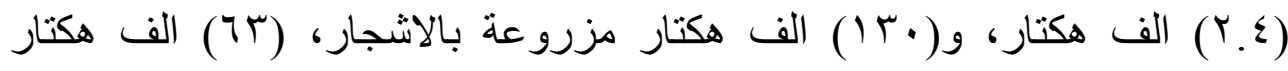

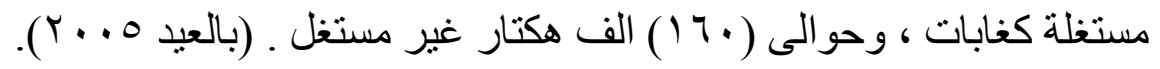


جدول رقم (1) : يوضح انتاج العنب فى بعض دول الوطن العربي خلال الفترة

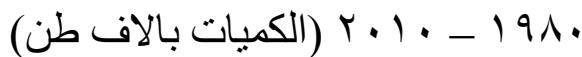

\begin{tabular}{|c|c|c|c|c|c|c|c|c|c|c|c|}
\hline \multirow[t]{2}{*}{ الاجمالي } & \multicolumn{10}{|c|}{ الاولة } & \multirow[t]{2}{*}{ السنة } \\
\hline & ليبيا & اليمن & الجزائر & تونس & السعودية & لبنان & المغرب & العراق & سوريا & مصر & \\
\hline $1 \leqslant \leqslant 0$ & $1 \varepsilon$ & 07 & TY & $1 \cdot 1$ & 77 & $1 \leq$. & 187 & 109 & 140 & r१q & $\Lambda$. \\
\hline 1071 & rq & $7 \varepsilon$ & $T V$ & 1.0 & 70 & 10. & 187 & $r .1$ & $\varepsilon .9$ & $r q 1$ & (1) \\
\hline 1870 & 10 & 71 & $T Y$ & 91 & $\varepsilon \varepsilon$ & 171 & TI. & YO. & $\varepsilon r r$ & $r .7$ & NT \\
\hline ITAY & YI & VY & $\vee \wedge$ & 1.0 & $\varepsilon Y$ & IVT & r.. & rq. & $r \wedge \Lambda$ & $r \leqslant \varepsilon$ & $\Lambda T$ \\
\hline$Y \cdot \leqslant T$ & 19 & 1) & $\Lambda$. & $11 \mathrm{r}$ & VV & 17. & $r \ldots$ & $\leqslant 7$. & $\varepsilon \ldots$ & rov & $\wedge \varepsilon$ \\
\hline YIVI & $r$. & $\Lambda$. & $1 \wedge r$ & ir. & $7 V$ & 10. & YTI & $\varepsilon .9$ & $\sum \wedge T$ & r90 & 10 \\
\hline 1119 & Y) & $1 . r$ & 91 & 110 & NT & 101 & $\mu \cdot \Lambda$ & r\^ & $0 \ldots$ & $r \leqslant Y$ & 17 \\
\hline r 100 & YY & 149 & $11 \mathrm{r}$ & 111 & $1 \ldots$ & 17. & Tr. & ror & $\varepsilon r r$ & 01. & $\Lambda \mathrm{V}$ \\
\hline$r \leqslant ा r$ & $T M$ & ITr & ITE & 91 & $\wedge$. & r). & $19 V$ & TI & ov1 & $00 \mathrm{~V}$ & $\Lambda \wedge$ \\
\hline rAYo & ro & 1 To & $|r|$ & $\Lambda \leqslant 1$ & $9 \vee \wedge$ & YIV & Y77 & $r \cdot \varepsilon$ & $\varepsilon \cdot V$ & TrI & 19 \\
\hline$r \ldots r$ & ד & $1 \leqslant Y$ & ITO & VVT & 1.1 & $r \wedge$. & TMY & $r \S 1$ & $\varepsilon r r$ & $79 \varepsilon$ & 9 . \\
\hline$r \wedge . r$ & rᄉ & $1 \pi \wedge$ & $r .1$ & $9 \leqslant V$ & or & rAo & 110 & YOY & $\varepsilon \wedge V$ & ד4 & 1991 \\
\hline דצ & rA & $1 \leq 0$ & Tr. & $9 V$. & 1.9 & rד & rqะ & $\varepsilon \cdot \Lambda$ & 纟Tr & 701 & 1994 \\
\hline$r \leq Y V$ & $r$. & $1 \leq \varepsilon$ & $r 10$ & 1.1 & Ir. & $r 09$ & Tr. & $1 \leq 1$ & ros & $V Y T$ & 1994 \\
\hline rYVA & $r$. & $1 \leq 7$ & 197 & $1 . r$ & $T K \varepsilon$ & rYA & TAY & TAY & rזr & $V \cdot V$ & $199 \varepsilon$ \\
\hline 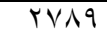 & $r$. & 10. & $19 V$ & 1.0 & 149 & $r \leqslant q$ & IYA & OVA & rAs & $V r q$ & 1990 \\
\hline$r V \cdot r$ & T & 91 & 197 & $\Lambda \wedge$. & $1 T K$ & $r T \leq$ & IYA & $\leqslant 10$ & $0 \leq$. & $9 \leqslant r$ & 1997 \\
\hline rAVO & rᄉ & 10. & 194 & 1.9 & $1 \leqslant$. & ros & $T \cdot \varepsilon$ & rqג & $\leqslant O Y$ & ᄉฯ^ & $199 V$ \\
\hline$r \ldots r$ & ru & 100 & $1 \leqslant V$ & 1.0 & $1 \leq 1$ & rNI & 519 & $r V \leq$ & 09. & 901 & 1991 \\
\hline rorq & $r \Lambda$ & 100 & $1 \vee \lambda$ & $1 . r$ & 974 & 99 & $\sum r T$ & $T V \leq$ & rNV & $1 \ldots 9$ & 1999 \\
\hline rvo. & 0. & 107 & $r \cdot r$ & $1 \leqslant r$ & $11 \mathrm{~V}$ & $11 r$ & $r \cdot V$ & $T V V$ & $\Sigma 1$. & 1.10 & $r \ldots$ \\
\hline rTr & $\varepsilon$ & 174 & YI & $1 \leq 0$ & 911 & 117 & $19 Y$ & $r \ldots$ & r19 & $1 \cdot V \wedge$ & $r \ldots l$ \\
\hline rosV & $r$. & $17 \varepsilon$ & $T T \varepsilon$ & 111 & 9 9Y० & $1 . r$ & TYV & ro & $r \leqslant r$ & $1 \cdot v \varepsilon$ & $r \ldots r$ \\
\hline rVVT & $\mu$. & $17 V$ & $r \vee \wedge$ & $1 . V$ & $9 \mathrm{qV}$ & 119 & $r \wedge I$ & r ro & $r \cdot v$ & 1198 & $r \ldots r$ \\
\hline rI. $\varepsilon$ & $\mu$. & $1 \cdot \leq$ & $r \wedge \varepsilon$ & ITV & $11 \varepsilon$ & IrT & TAS & $r$. & $r \leqslant r$ & ITVO & $r \ldots \varepsilon$ \\
\hline$r .0 \mathrm{~V}$ & $r$. & 1.1 & TTE & TKY & ITK & 111 & TrV & 110 & $r . \tau$ & 1149 & $r \ldots o$ \\
\hline rrvq & $r$. & $11 \mathrm{~V}$ & $r 91$ & ITr & rVo & 11. & rד & 110 & TrV & $1 \leqslant r r$ & $r \ldots T$ \\
\hline r. ro & $r$. & $1 Y 7$ & $r \leqslant 0$ & $1 . Y$ & $1 \leq \varepsilon$ & 11. & דו & $1 \wedge \varepsilon$ & TVT & $1 \leqslant \wedge 0$ & $r \cdots V$ \\
\hline ITrT & $r \cdot$ & ITV & $\varepsilon \cdot 1$ & IYY & 174 & 111 & $r \leqslant 7$ & $r \cdot r$ & $r \wedge 1$ & 1041 & $r \cdots \Lambda$ \\
\hline TMYA & Tr & 119 & $\sum 94$ & $1 \pi$ & 17. & $1 Y$. & TrA & 190 & rod & $1 T V$. & $r \ldots q$ \\
\hline ז' & ro & 177 & 07. & 119 & $1 \pi N$ & $|r|$ & 417 & $r / T$ & rYT & $11 \%$. & $r .1$. \\
\hline & $r$. & $1 \% 0$ & $r .9$ & rrq & r4r & 199 & YOs & $r \wedge \varepsilon$ & $\varepsilon \ldots$ & $\wedge 0$. & متوسط \\
\hline
\end{tabular}

المصدر: المنظمة العربية للتنمية الزر اعية ، الكتاب السنوي للاحصائيات الزر اعية ــ الخرطوم

$$
\text { - السودان-اعداد متفرقة. }
$$

ويعتبر انتاج ليبيا من العنب منخفضا مقارنة مع الدول العربية حيث تأتى فى لقى

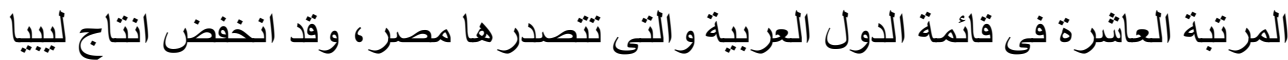

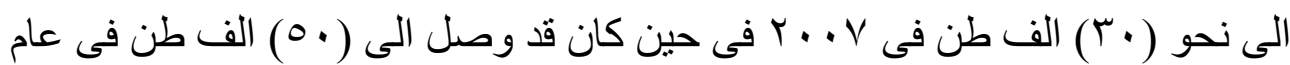
(Y... 
ولوحظ تذبذب المساحة المزروعة من العنب فى ليبيا خلال فترة الدراسة،

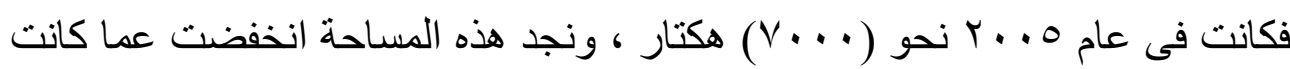

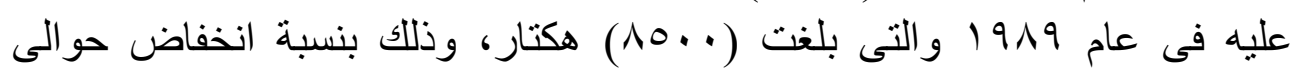

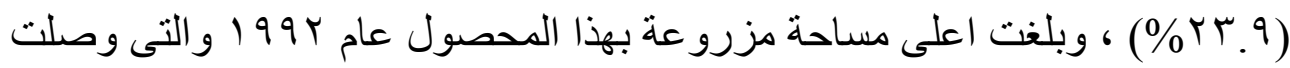

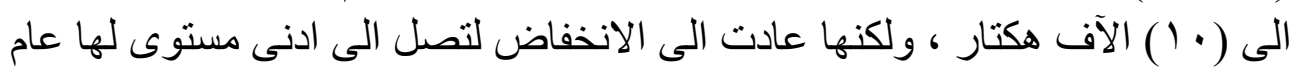

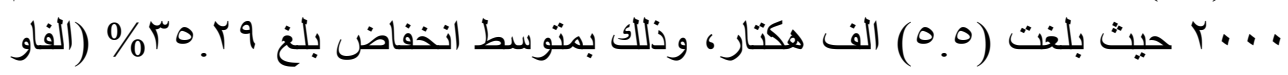

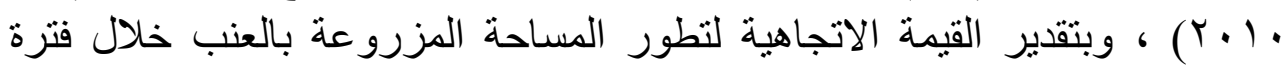

$$
\begin{aligned}
& \text { الدراسة نجد ان افضل صورة هي المعادلة الاسية النالية:- } \\
& e=y^{r} \cdot r \cdot \cdots \cdot t_{t} \\
& \mathrm{~T}=(r \Upsilon . \varepsilon)(-r . \wedge) \quad \mathrm{R}^{r}=\cdot . \mathrm{\Lambda}
\end{aligned}
$$

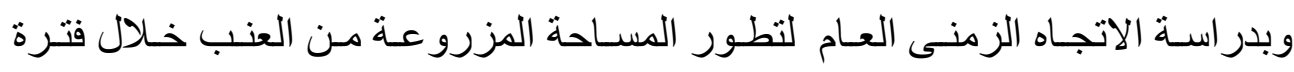

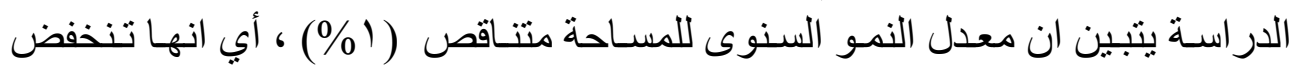

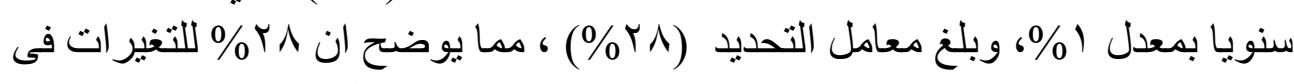

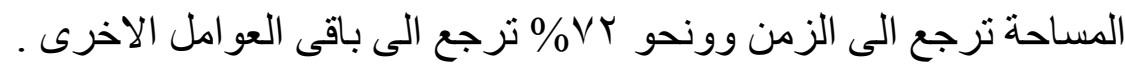

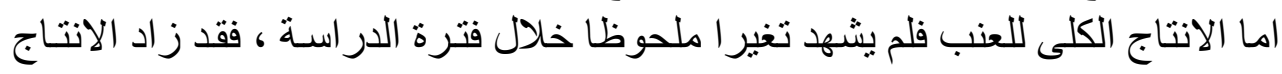

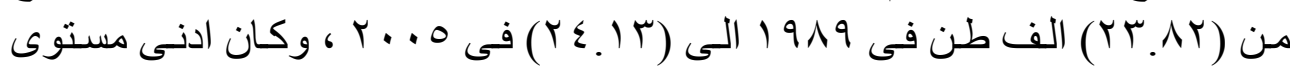

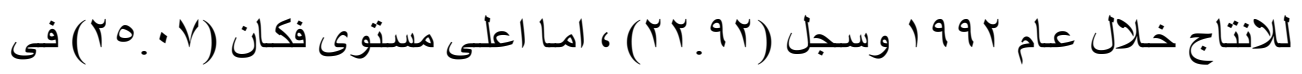

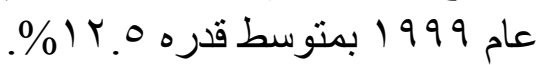
وبتقدير دالة اتجاه الزمن العام مع الانتاج الكلى كانت الصورة كما فى المعادلة التالية:

$$
\begin{gathered}
\mathrm{y}=\mathrm{e}^{r \cdot 10+\cdot . \cdot r_{\mathrm{t}}} \\
\mathrm{T}=(r \varepsilon \cdot)(r .0 \mathrm{~V}) \quad \mathrm{R}^{r}=\cdot r^{r q}
\end{gathered}
$$

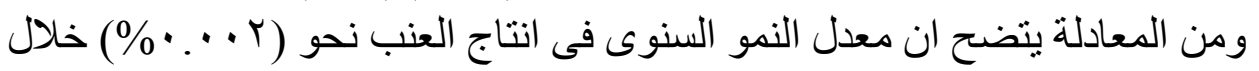

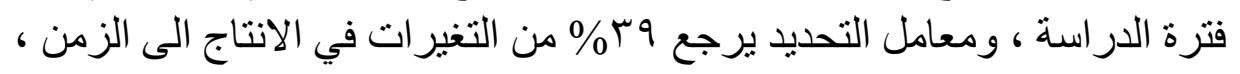
و آT\% الى باقي العوامل الاخرى.

\section{تقدير تطور انتاجية العنب فى ليبيا}

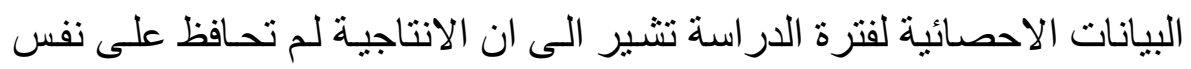

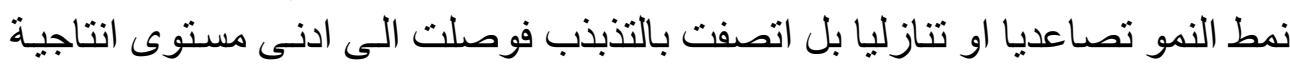

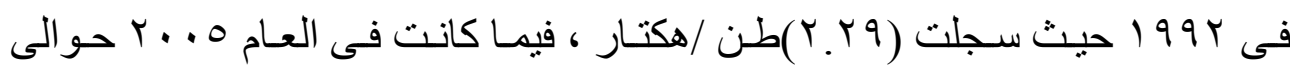

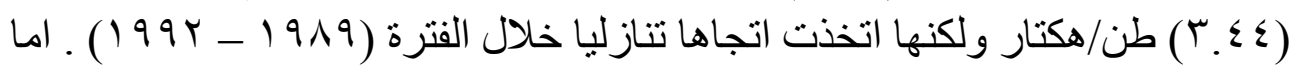




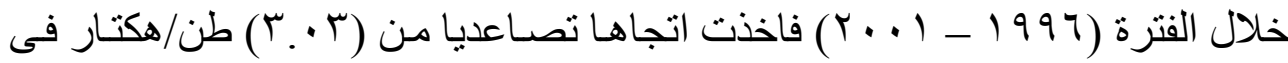

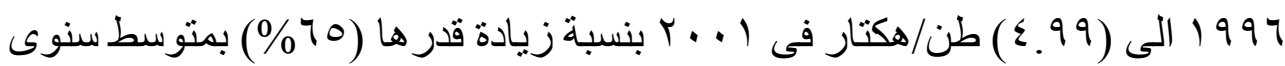

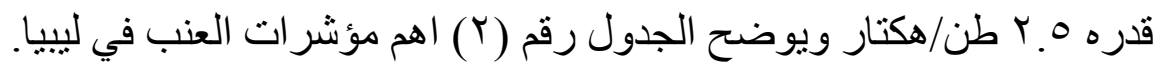

بتقدير القيمة الاتجاهية لتطور انتاجية العنب كاتت افضلها الصور التالية: $\mathrm{G}=\mathrm{e}^{0.94+0.001 \mathrm{t}}$

$$
\mathrm{T}=(l r . r)(\cdot r r) \quad \mathrm{R}^{r}=\cdot r r
$$

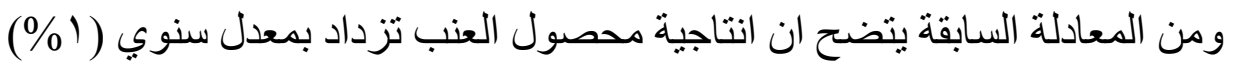

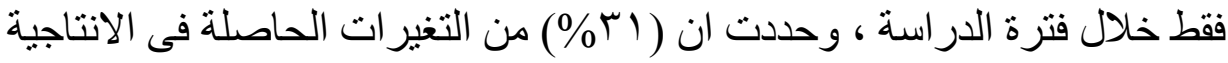

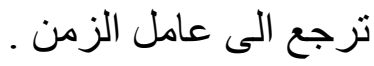

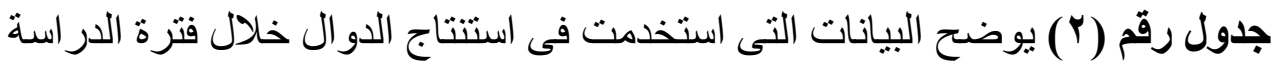

\begin{tabular}{|c|c|c|c|c|c|}
\hline (درجة مئوية) & (بالاينار الليبي) & طن/هتارية & 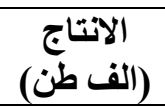 & المساحة الف & السنوات \\
\hline TO & $T A Y$ & r.A. & TY.AY & 1.0 & 1919 \\
\hline YT & VI. & Y.TE & $r T . V V$ & 9 & 199. \\
\hline$T V$ & 94. & $r . \leqslant T$ & $r T . \varepsilon r$ & 9.0 & 1991 \\
\hline TA & Vol & r.rq & Tr.90 & 1. & 1995 \\
\hline YT & THA & Y.OV & YT.VT & $9 . Y$ & 1994 \\
\hline ru & $7 \wedge V$ & Y.OT & Tr.YO & Q.YY & $199 \leqslant$ \\
\hline TV & A.. & $r . \leq \varepsilon$ & $r T .0$ & 9.7 & 1990 \\
\hline ro & 1.0. & $r . r$ & $r T .7 \Lambda$ & V. 1 & 1997 \\
\hline YT & $1 \varepsilon \ldots$ & $r . \wedge$ & $r T . \wedge V$ & $V .0$ & $199 \mathrm{~V}$ \\
\hline Y0.7 & $1 \% \ldots$ & $r . Y \xi$ & $T \Sigma . T V$ & 9.01 & 1991 \\
\hline$r \leqslant . r$ & $10 \ldots$ & ז.7. & YO.V & 7.97 & 1999 \\
\hline YY. & $1 \leqslant \Lambda$. & $\varepsilon . T_{T}$ & $r T . \wedge 1$ & 0.0 & $r \ldots$ \\
\hline$r \leq .0$ & $1 \% \ldots$ & $\varepsilon .99$ & $r \leqslant .00$ & 7 & $r \ldots l$ \\
\hline YT & $10 \ldots$ & r.l. & $r \leqslant . \wedge 0$ & $\wedge$ & $r \ldots r$ \\
\hline rq & 170. & r.VT & $r T . \leqslant \Lambda$ & 1.0 & $r \ldots r$ \\
\hline YT & $1 \pi \mid$. & $r . r \mu$ & $T \Sigma . Y T$ & $V .0$ & $r \ldots \varepsilon$ \\
\hline Y & $17 \ldots$ & r. $\leqslant \varepsilon$ & $r \leq .1 T$ & V & $r \ldots o$ \\
\hline TV & 100. & $r . \leqslant 0$ & $Y \leqslant . Y$ & V & $r \ldots r$ \\
\hline Yo.0 & 101. & r.7. & Tr.A & 7.7 & $r \ldots v$ \\
\hline
\end{tabular}




\begin{tabular}{|c|c|c|c|c|c|}
\hline rA & 171. & $r . \leqslant 0$ & $Y \leqslant . Y$ & V & $r \ldots \Lambda$ \\
\hline$r 7$ & 187. & r.r. & $r \leqslant . \wedge$ & $V .0$ & $r . . q$ \\
\hline TV & $I V V$. & $r .1$ & ro.7 & 1.0 & $r \cdot 1$. \\
\hline YY.Y & IYOY.IA & 1.10 & $Y \varepsilon \ldots \varepsilon$ & $V . \varepsilon$ & المتوسط \\
\hline
\end{tabular}

\section{بناء وتقدير النموذج القياسى لدالة استجابة عرض العنب:}

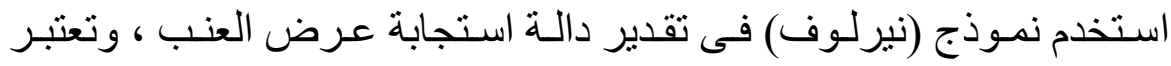

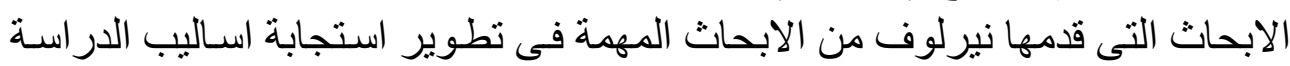

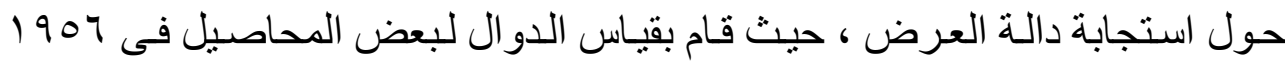

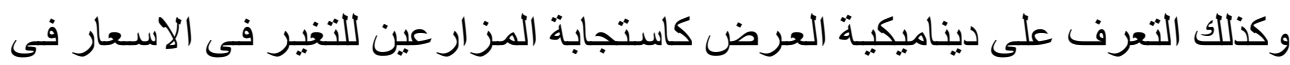

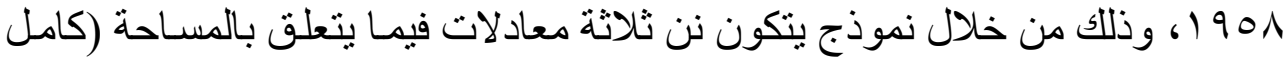

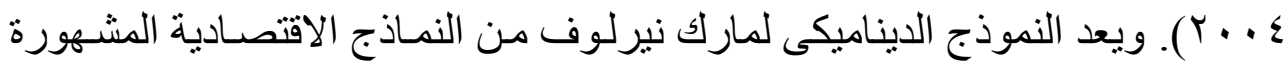

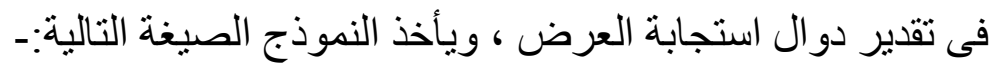

$$
\mathrm{Y}_{\mathrm{t}}^{*}=\alpha \mathrm{xb}_{\mathrm{t}-1}+\mathrm{tM}
$$

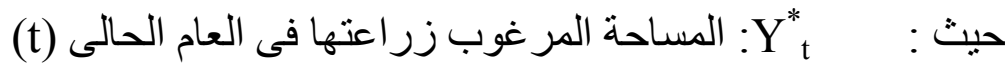

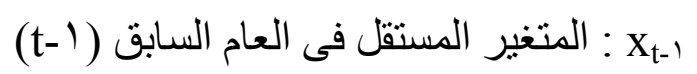

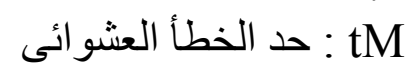

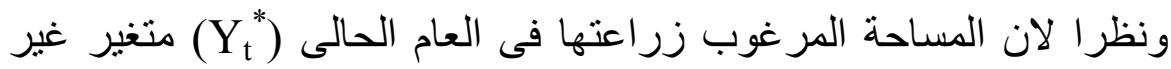

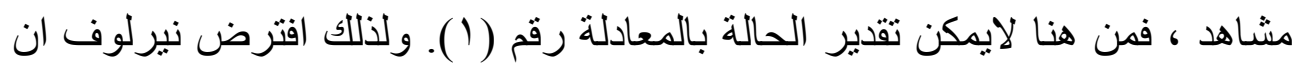

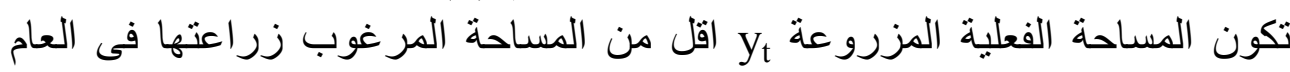

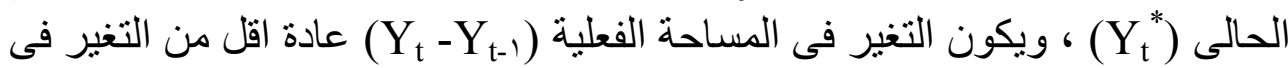

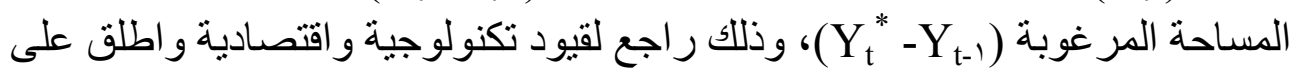

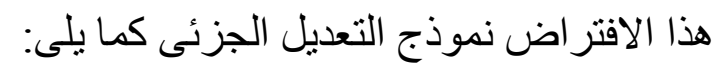

$$
\left.\left.\left(\mathrm{Y}_{\mathrm{t}}-\mathrm{Y}_{\mathrm{t}-1}\right)=\mathrm{Y}\right) \chi_{\mathrm{t}}^{*}-\mathrm{Y}_{\mathrm{t}-1}\right)
$$

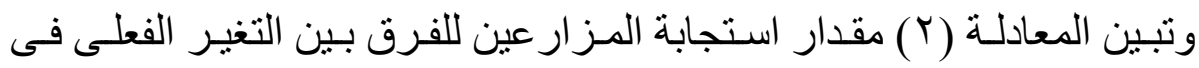

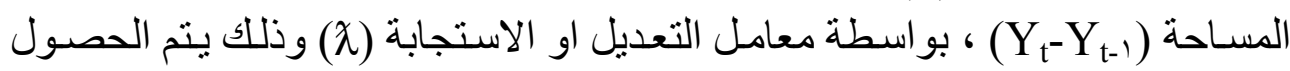


$\mathrm{Y}_{\mathrm{t}}=\mathrm{Y} \chi_{\mathrm{t}}^{*}+\left(1-\mathrm{Y}\left(\chi_{\mathrm{t}-1}\right.\right.$

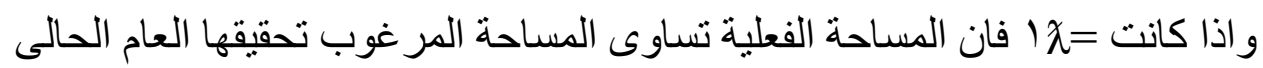
اى (y

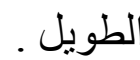

اما اذا كانت (=x • ) فان المساحة الفعلية فى العام الحالى تساوى المساحة الفعلية فى

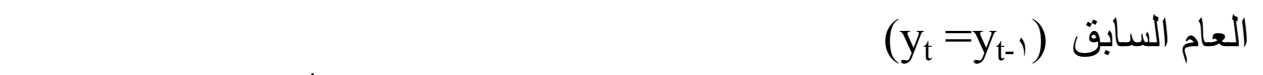

$$
\left.\mathrm{y})=\chi_{\mathrm{t}}-\mathrm{y}_{\mathrm{t}-1}\right) /\left(\mathrm{y}_{\mathrm{t}}^{*}-\mathrm{y}_{\mathrm{t}-1}\right)
$$

وتتشير المعادلة السابقة الى مقدار التغير فى المساحة المر غوبة نسبة الى مقار التغير فى المساحة الفعلية ، وبحساب الفترة الزمنية لحدوث العير الاستجابة الكاملة :

$\chi=\left(\mathrm{y}_{\mathrm{t}}^{*}-\mathrm{y}_{\mathrm{t}-1}\right) /\left(\mathrm{y}_{\mathrm{t}}-\mathrm{y}_{\mathrm{t}-1}\right)=(1 \lambda /)$

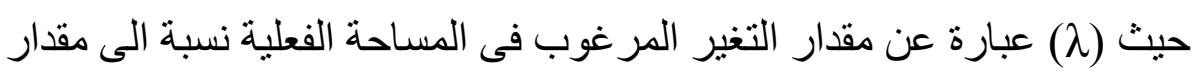

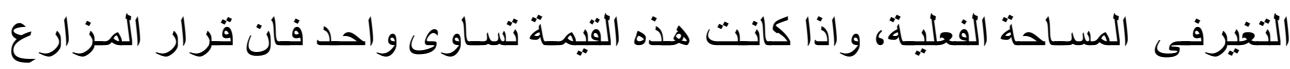

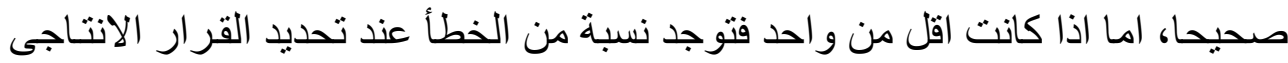

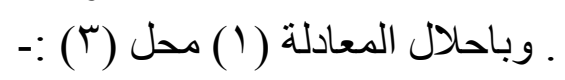

$\mathrm{y}_{\mathrm{t}}=\mathrm{a} \lambda \mathrm{x}_{\mathrm{t}-1}+(1-\lambda) \mathrm{y}_{\mathrm{t}-1}+\mathrm{M}_{\mathrm{t}}$

حيث ان :-

(t) =y

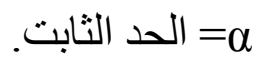

$$
\text { = معامل الانحدار. }
$$

•

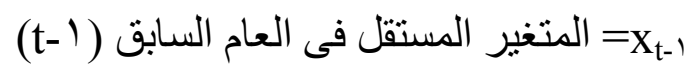

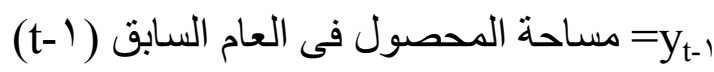

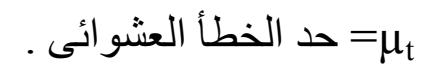

وبافتر اض ان دالة الانحدار اللازمة لتقديردالة استجابة العرض كالتالى :

$\mathrm{y}_{\mathrm{t}}=\alpha .+\alpha, \mathrm{X}_{\mathrm{t}-1}+\alpha_{\mathrm{r}} \mathrm{y}_{\mathrm{t}}+\mu_{\mathrm{t}}$

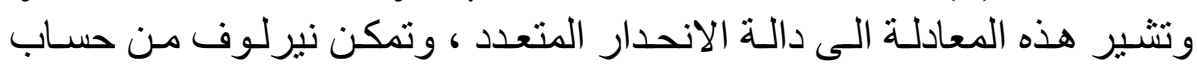

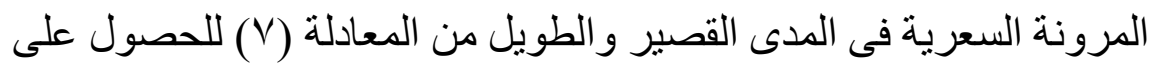




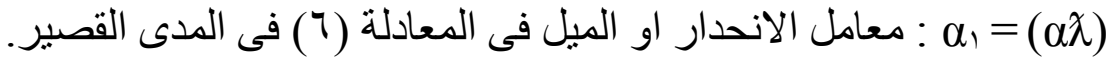

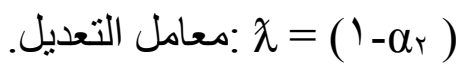

ون

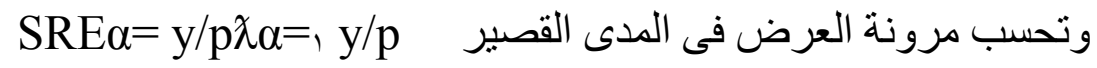
اما فى المدى الطويل

سيكون تقدير دالة استجابة العرض فى هذه الدراسة بالصورة التالية : $\left.\mathrm{y}_{\mathrm{t}}=\mathrm{P}\right) \mathrm{F}_{\mathrm{t}-1}$ ' $\mathrm{A}_{\mathrm{t}}$ 'T $6 \mathrm{TMP}_{\mathrm{t}}\left(\mathrm{y}_{\mathrm{t}-1}\right)$ ).

\section{وصف النموذج :}

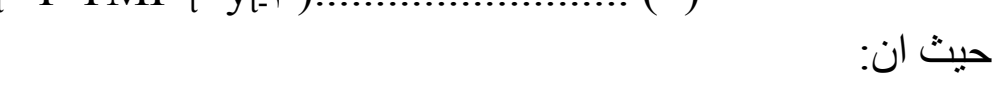

الانتاج الكلى من العنب فى السنة (t) =yt

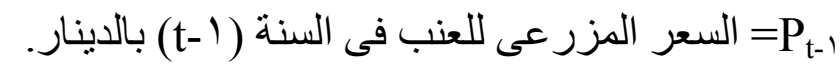

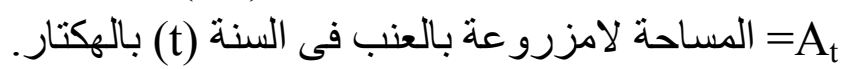
متغير زمنى يوضح التكنولوجيا الانتاجية فى السنة (t) بالسنة. درجات الحر ارة لنمو المحصول في السنة (t) درجة مئولية.

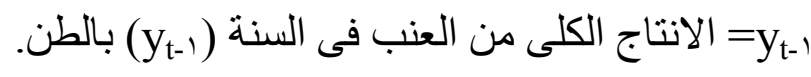

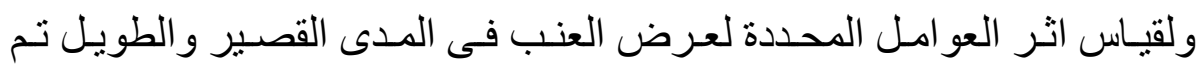

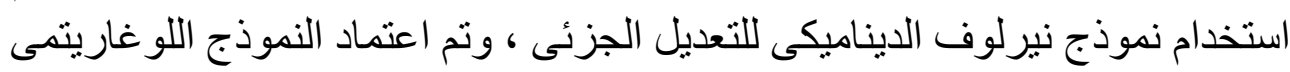

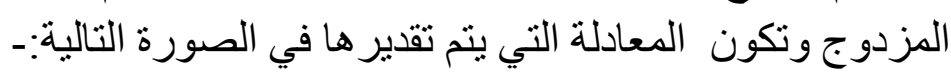
y nl $=\alpha . \chi+\alpha, p n l \chi_{t-1}+\alpha_{r}$ T nl $\chi_{1}+\alpha_{r}$ PMTnl $\chi_{t}+\left(1-y n l\left(\chi_{t}-1\right.\right.$ e +

\section{تقدير دالة استجابة العنب عرض العنب :-}

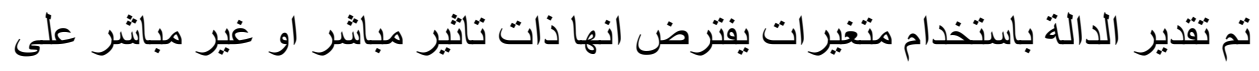

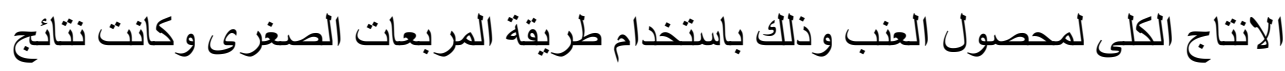

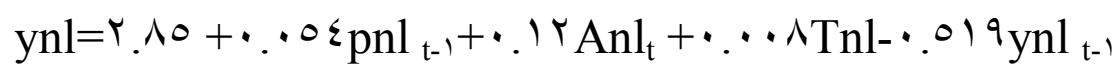

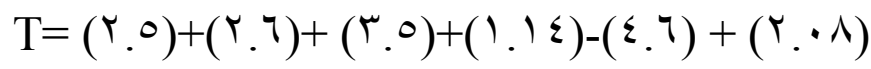

$$
\begin{aligned}
& \mathrm{R}^{r}=.79 \mathrm{~F}=\wedge
\end{aligned}
$$


تثير المعادلة الى وجود علاقة طردية بين كل من المتغيرات المستقلة التالية

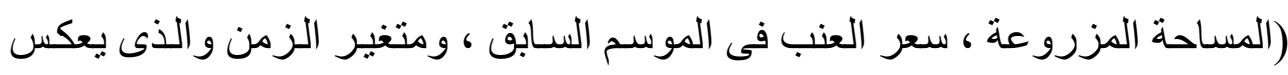

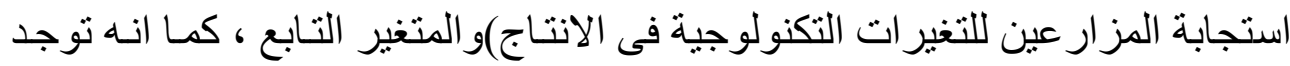

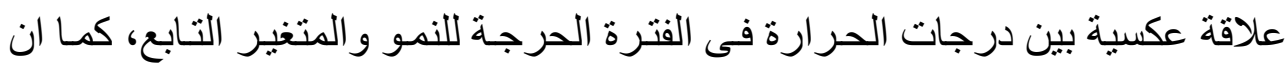

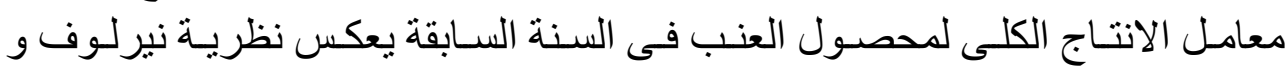

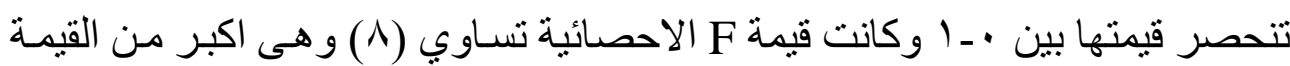

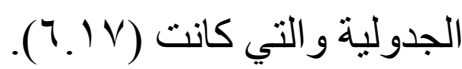

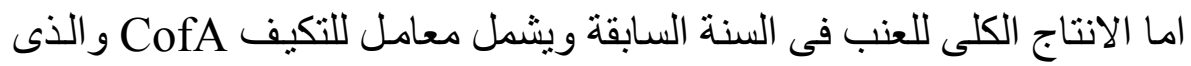

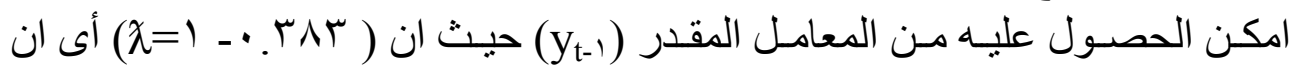

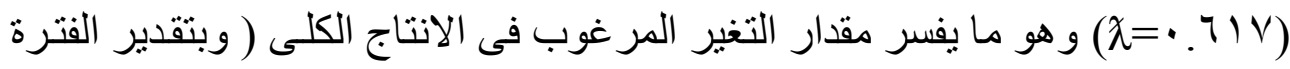

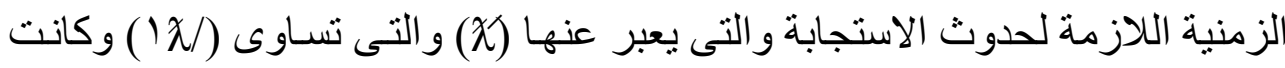

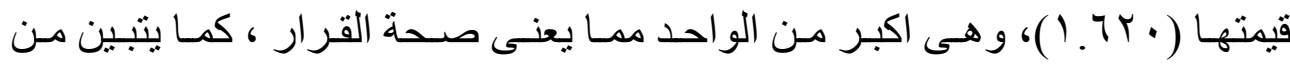

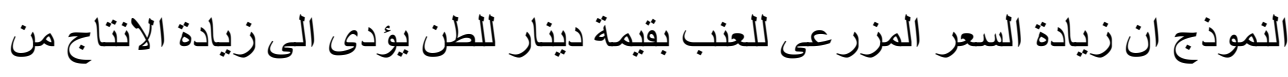

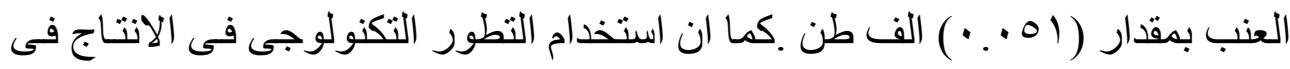

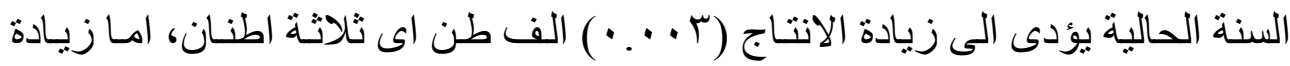

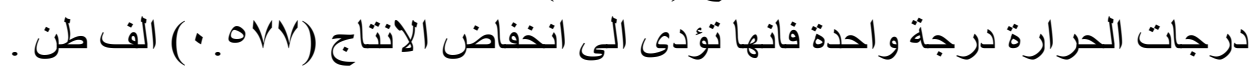

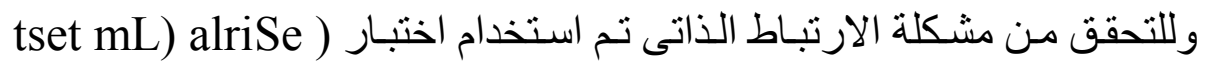

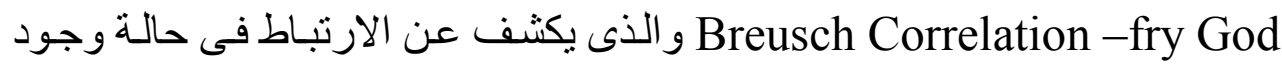

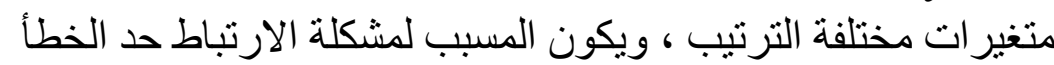
F-static $=\cdot . \leqslant 9(\cdot .7 r)$

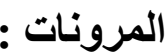

وتم احتسـاب مرونـة العرض السعرية لمحصول العنب فى المدى القصير باستخدام

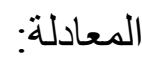

$$
\left.\ln y \partial)=\partial_{\mathrm{t}} \operatorname{lnp} \partial / \mathrm{t}^{-1}\right) *\left(\ln \mathrm{p}_{\mathrm{t}-1} / \ln \mathrm{y}_{\mathrm{t}}\right)
$$

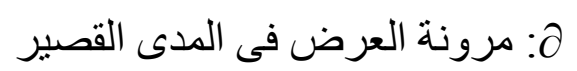

$$
\text { حيث ان : }
$$

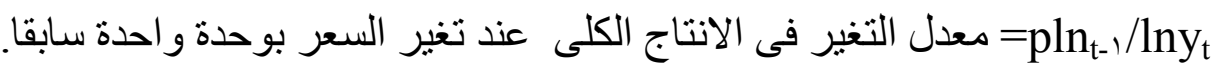

$$
\text { = lnpt-1 }
$$

= lnyt 
ومن خلال النموذج السابق تكون مرونة العرض فى المدى القصبر كالتالى:

$$
\text { S.R.E. }=* .0 \leq *(Y .9 r 0 / 1 . r 17)=. .1 r \text {. }
$$

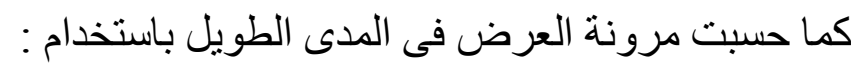

$\mathrm{SRE})=\mathrm{E} \cdot \mathrm{R} \cdot \mathrm{L} \lambda /)=(\cdot .1 \mathrm{r} \cdot / \cdot .7 / \mathrm{V})=\cdot 19 \varepsilon$

التتائج والتوصيات:

اظهرت النتائج ان استجابة محصـول العنب لتغير السـرتعد ضـعيفة وهو مـا

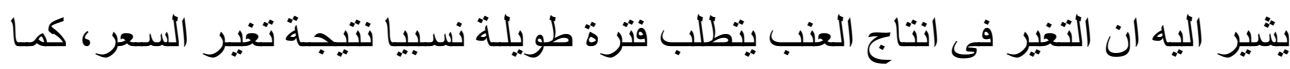

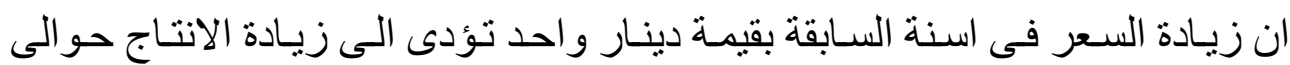

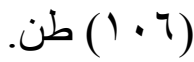

وقد اوضـحت النتائج ان لارتفـاع درجات الحرارة علاقة عكسية على الانتـاج الامر الذى يؤثر سلبا على عرض العنب فى المدى القصير.

\section{وتوصى الاراسة من خلال التتائج بالآتى:}

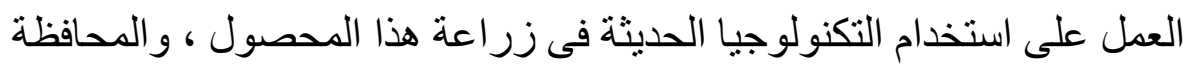

على المساحات المزرو عة منه، و التوسع فيها وايجاد اصناف عالية الانتاجية.

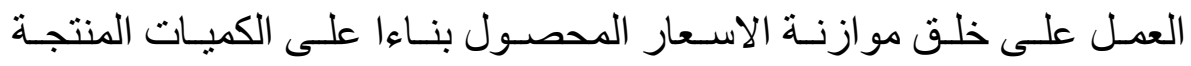

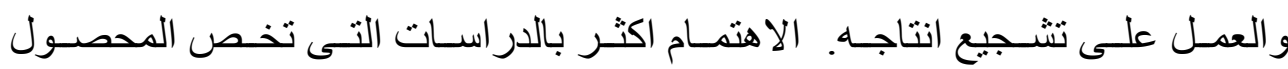

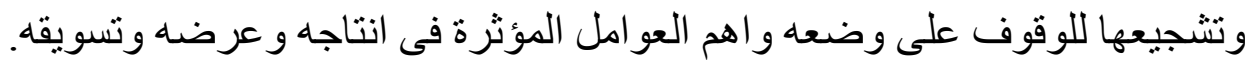

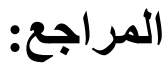

• محمد علاعالاين كامل " الاساليب البحثية الحديثة فى در اسة السياسات

السعرية" ، ماعلة القاهرة

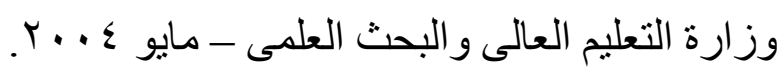

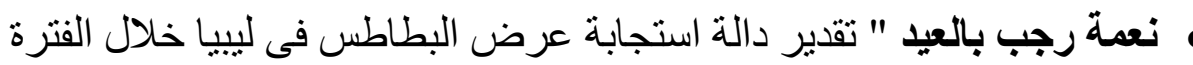

$" r \ldots r-19 V$

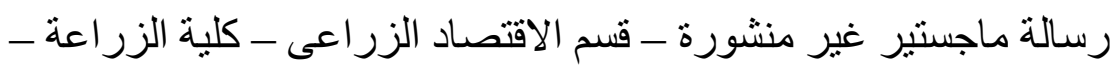

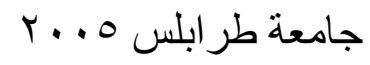




$$
\begin{aligned}
& \text { • منظمة الاغذية و الزر اعة (الفاو) الكتاب السنوى - اعداد مختلفة - روما. }
\end{aligned}
$$

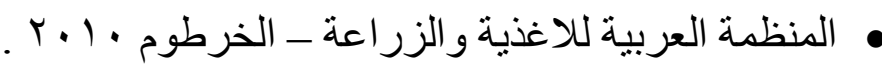

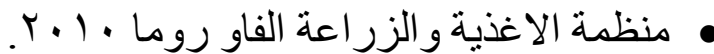

\title{
ASSESSMENT OF SUPPLY IN RESPONSE TO THE GRAPE HARVEST IN LIBYA DURING THE PERIODS (1990-2010)
}

\author{
Ahmed Mabrouk El Sadek* and Mohamed Yuseef Bin-Isa** \\ * Assistant professor, Faculty of Agriculture, University Sabha, Libya \\ **Lecturer of Faculty of Agriculture, University of Tripolis, Libya.
}

\section{ABSTRACT}

This study aims to identify the most important factors that affect the display grape crop response, assessment of supply function using a number of variables and flexibility account, during the period (19902010), and used the logarithmic function in the function the estimate using the model Marc Narlov dynamic.

The results showed that the most important determinants of the grapes display is the price and the area planted and technological development, and the results showed that the total production of grapes in response to the price change in the short and long term that it is flexible.

Key words: Assessment of supply, response to the grape harvest, Libya. 